\title{
Os hidrocarbonetos e a teoria dos grafos
}

\author{
Roberto Ribeiro Paterlini(10 Romeu C. Rocha-Filho®i
}

\begin{abstract}
Resumo
Na disciplina de Química do Ensino Médio os estudantes aprendem o que são os hidrocarbonetos, uma extensa e importante família de compostos orgânicos. A estrutura combinatória dessas moléculas pode ser modelada por grafos, e alguns resultados de fácil compreensão desse objeto matemático traduzem propriedades daqueles compostos. Vemos, assim, uma oportunidade para os professores de Química e Matemática praticarem a interdisciplinaridade. O professor de Matemática, em particular, pode aproveitar essa inter-relação para abordar os grafos, uma importante área da Matemática Discreta. Neste texto, pretendemos fornecer informações para a construção de sequências didáticas com esse objetivo.
\end{abstract}

Palavras-chave: Grafos; árvores; modelos de hidrocarbonetos; contagem de átomos de hidrogênio; isômeros.

\begin{abstract}
In high school chemistry, students learn of hydrocarbons, an extensive and important family of organic compounds. The combinatorial structure of these molecules can be modeled by graphs, and some easy-to-understand results of that mathematical object render properties of those compounds. Thereby we see an opportunity for chemistry and mathematics teachers to practice interdisciplinarity. Mathematics teachers, in particular, can take advantage of this interrelation to approach graphs, an important area of Discrete Mathematics. In this article, we intend to provide information for the construction of didactic sequences with that purpose.
\end{abstract}

Keywords: Graphs; trees; hydrocarbon models; count of hydrogen atoms; isomers.

\section{Introdução}

Os hidrocarbonetos são compostos orgânicos formados somente por átomos de carbono e hidrogênio. Neste artigo, focaremos, em três tipos básicos de hidrocarbonetos, os alcanos, os alcenos e os alcinos. O que distingue os alcanos dos outros tipos de hidrocarbonetos é que em suas moléculas os átomos de carbono sempre estão ligados a quatro outros átomos, sejam eles de carbono e/ou hidrogênio, sendo que as ligações carbono-carbono sempre são simples. Como quatro é o número máximo de ligações que um átomo de carbono pode fazer, os alcanos também são conhecidos como hidrocarbonetos saturados. Por outro lado, existem dois subtipos de alcanos: os acíclicos (comumente referidos simplesmente como alcanos) e os cíclicos (referidos como cicloalcanos ou naftenos). Em alguns hidrocarbonetos, existem ligações carbono-carbono que são duplas ou triplas, isto é, 
existem átomos de carbono que estão ligados a somente três ou dois outros átomos. Quando existe uma ligação carbono-carbono dupla, o hidrocarboneto é conhecido como alceno. No caso de uma ligação carbono-carbono tripla, o hidrocarboneto é um alcino. Tanto os alcenos como os alcinos são conhecidos como hidrocarbonetos insaturados, pois suas moléculas têm dois átomos de carbono que estão ligados a menos de quatro outros átomos, sejam eles de carbono e/ou hidrogênio. Como no caso dos alcanos, também existem subtipos cíclicos dos hidrocarbonetos insaturados.

Sob o ponto de vista combinatório, as moléculas desses diferentes hidrocarbonetos podem ser modeladas por um objeto matemático chamado grafo. Para os diferentes tipos de hidrocarbonetos, alguns teoremas muito simples permitem-nos calcular o número de átomos de hidrogênio de uma molécula em função do número de átomos de carbono. Surpreendentemente, essas relações são fixas, embora as estruturas das moléculas possam variar muito.

\section{A espetacular família dos alcanos acíclicos}

Uma propriedade que chama a atenção nas moléculas dos alcanos acíclicos é que, se o número de átomos de carbono é $n$, o de hidrogênio é sempre $2 n+2$, qualquer que seja a estrutura molecular formada pelos $n$ átomos de carbono. Assim, em Química, a fórmula geral dos alcanos acíclicos é $\mathrm{C}_{\mathrm{n}} \mathrm{H}_{2 \mathrm{n}+2}$. Veremos como alguns teoremas sobre grafos permitem-nos obter essa relação.

Nos alcanos acíclicos cujas moléculas têm quatro ou mais átomos de carbono, esses podem estar ligados formando estruturas lineares, cada átomo de carbono ligando-se a no máximo dois outros átomos de carbono, ou estruturas ramificadas, quando também existem átomos de carbono que se ligam a outros três ou quatro átomos de carbono. Com isso, surge a possibilidade de diferentes estruturas moleculares para uma mesma fórmula molecular, dando origem a diferentes compostos, conhecidos como isômeros. Por exemplo, para a fórmula molecular $\mathrm{C}_{5} \mathrm{H}_{12}$, existem três isômeros (Figura 1), um com estrutura linear (referido como pentano) e dois com estruturas ramificadas (referidos como isopentano e neopentano). Na molécula do isopentano, há um átomo de carbono ligado a três outros átomos de carbono, e na do neopentano, o átomo central está ligado aos outros quatro átomos de carbono. Embora as estruturas das três moléculas sejam diferentes, continua valendo a relação de $2 \mathrm{n}+2$ átomos de hidrogênio para $\mathrm{n}$ átomos de carbono.
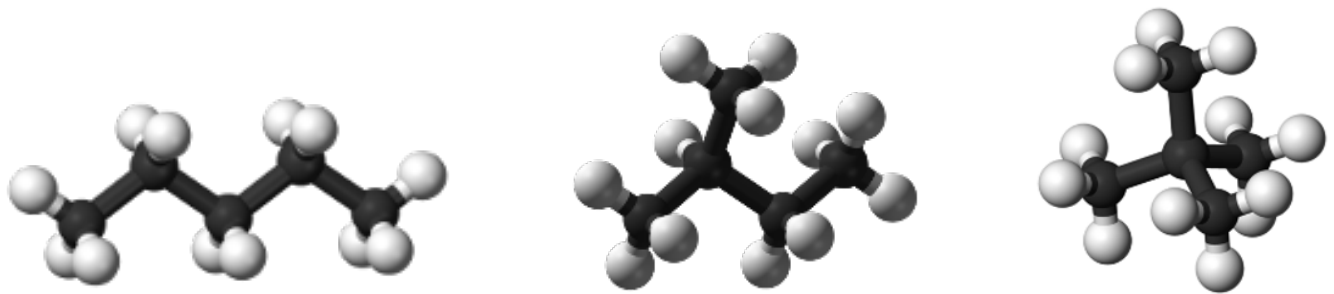

Figura 1: Ilustração dos três isômeros de fórmula molecular $\mathrm{C}_{5} \mathrm{H}_{12}$, conhecidos como pentano, isopentano e neopentano. Autores: Ben Mills e Jynto [8].

\section{A família dos cicloalcanos}

Os cicloalcanos são hidrocarbonetos saturados que contêm um único ciclo em sua estrutura molecular, ao qual podem estar ligadas uma ou mais ramificações. Analogamente aos alcanos, nos cicloalcanos também há uma relação fixa entre o número de átomos de carbono e o de hidrogênio, que é $\mathrm{C}_{n} \mathrm{H}_{2 n}$, para $\mathrm{n} \geq 3$. Essa relação é igual à dos alcenos, hidrocarbonetos que contêm uma 
ligação dupla carbono-carbono, porém para $\mathrm{n} \geq 2$ (vide abaixo). Para a fórmula molecular $\mathrm{C}_{3} \mathrm{H}_{6}$, existe um único cicloalcano, o ciclopropano. Já para a fórmula $\mathrm{C}_{4} \mathrm{H}_{8}$, existem dois isômeros: o ciclobutano e o metilciclopropano, sendo esse um ciclopropano com uma ramificação, um grupo metil - veja a Figura 2. Para $\mathrm{n} \geq 5$, o número de isômeros aumenta bastante, pois surge um outro tipo de isômeros, os estereoisômeros, que decorrem da possibilidade de as ramificações assumirem diferentes posições relativas no espaço.
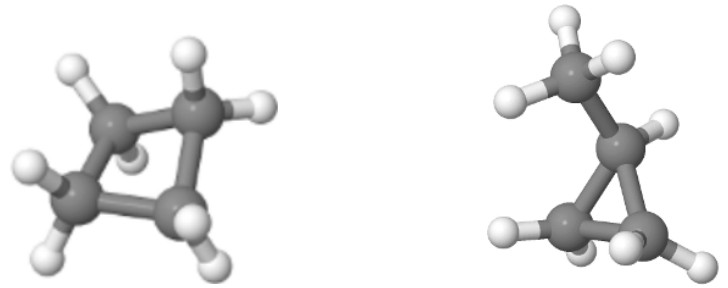

Figura 2: Ilustração dos dois cicloalcanos isoméricos de fórmula molecular $\mathrm{C}_{4} \mathrm{H}_{8}$, o ciclobutano e o metilciclopropano. Figuras geradas por [2].

Cabe destacar que, para um dado valor de n, a molécula do alcano sempre tem dois átomos de hidrogênio a mais que a correspondente molécula do cicloalcano. Assim, o cicloalcano, em condições adequadas (como no caso da presença de um catalisador de um metal de transição), pode ser hidrogenado, com a abertura do anel, transformando-se em um alcano. Por exemplo, o metilciclopropano $\left(\mathrm{C}_{4} \mathrm{H}_{8}\right)$ pode ser hidrogenado, obtendo-se o butano $\left(\mathrm{C}_{4} \mathrm{H}_{10}\right)$.

\section{Hidrocarbonetos insaturados: alcenos, cicloalcenos, alcinos e cicloalcinos}

Como já mencionado, os alcenos são hidrocarbonetos insaturados que têm uma ligação carbonocarbono dupla em suas moléculas e apresentam uma relação fixa entre o número de átomos de carbono e de hidrogênio igual à dos cicloalcanos: $\mathrm{C}_{\mathrm{n}} \mathrm{H}_{2 \mathrm{n}}$, para $\mathrm{n} \geq 2$. Para $\mathrm{n}=4$, existem quatro alcenos isoméricos: o 1-buteno, o cis-2-buteno, o trans-2-buteno e o isobuteno (ou isobutileno) veja a Figura 3. Nos três primeiros isômeros, há dois átomos de carbono ligados a dois outros átomos de carbono, enquanto no último isômero há um átomo ligado a três outros átomos de carbono. Note que esses quatro alcenos, de fórmula molecular $\mathrm{C}_{4} \mathrm{H}_{8}$, também são isômeros dos dois cicloalcanos mostrados na Figura 2. Portanto, existem seis isômeros para essa fórmula molecular. $\mathrm{O}$ alceno mais simples, o eteno (mais conhecido como etileno), $\mathrm{C}_{2} \mathrm{H}_{4}$, é o composto orgânico mais produzido industrialmente, com muitas aplicações. Por exemplo, a partir dele é obtido o polietileno, o plástico incolor mais usado mundialmente. Por outro lado, o eteno também é um importante hormônio natural de plantas, muito empregado para acelerar o amadurecimento de frutas.
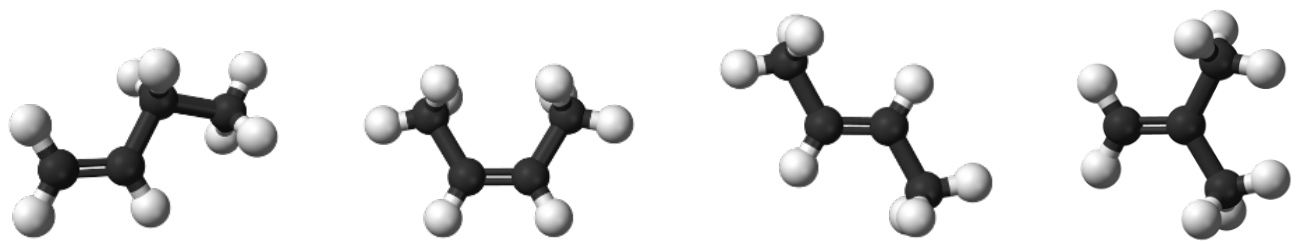

Figura 3: Ilustração dos quatro alcenos isoméricos de fórmula molecular $\mathrm{C}_{4} \mathrm{H}_{8}$ : a partir da esquerda, o 1-buteno, o cis-2-buteno, o trans-2-buteno e o isobuteno. Autor: Ben Mills [9]. 
Os cicloalcenos são hidrocarbonetos insaturados cuja estrutura molecular contém um ciclo com três ou mais átomos de carbono. A esse ciclo podem estar ligadas uma ou mais ramificações. Para esses compostos, a relação fixa entre o número de átomos de carbono e de hidrogênio é $\mathrm{C}_{\mathrm{n}} \mathrm{H}_{2 \mathrm{n}-2}$, para $\mathrm{n} \geq 3$. O mais simples deles é o ciclopropeno, $\mathrm{C}_{3} \mathrm{H}_{4}$. Para $\mathrm{n}=4$, a fórmula molecular é $\mathrm{C}_{4} \mathrm{H}_{6}$ e existem três cicloalcenos isoméricos, mostrados na Figura 4, na qual também é mostrado um alceno isomérico desses cicloalcenos.
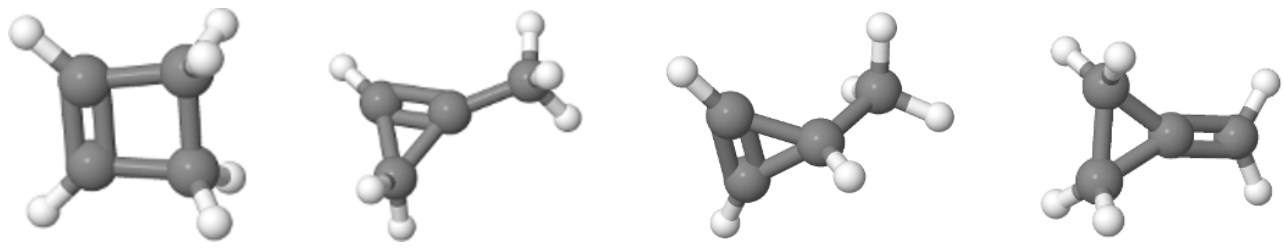

Figura 4: Ilustração dos três cicloalcenos isoméricos de fórmula molecular $\mathrm{C}_{4} \mathrm{H}_{6}$ : a partir da esquerda, o ciclobuteno, o 1-metilciclopropeno e o 3-metilciclopropeno. À direita, ilustração de um outro composto isomérico desses cicloalcenos, um alceno, o metilenociclopropano. Figuras geradas por [2].

Os alcinos são hidrocarbonetos insaturados que têm uma ligação carbono-carbono tripla em suas moléculas e apresentam uma relação fixa entre o número de átomos de carbono e o de hidrogênio igual à dos cicloalcenos, isto é, $\mathrm{C}_{\mathrm{n}} \mathrm{H}_{2 \mathrm{n}-2}$, para $\mathrm{n} \geq 2$. Portanto, o etino, $\mathrm{C}_{2} \mathrm{H}_{2}$, é o alcino mais simples. Para $\mathrm{n}=4$, existem dois alcinos isoméricos, mostrados na Figura 5. Note que esses dois alcinos, de fórmula molecular $\mathrm{C}_{4} \mathrm{H}_{6}$, também são isômeros dos quatro compostos mostrados na Figura 4. Portanto, as duas figuras exibem seis isômeros para essa fórmula molecular (na Wikipedia, ao se buscar pela fórmula molecular C4H6, são encontrados 10 compostos com essa fórmula).
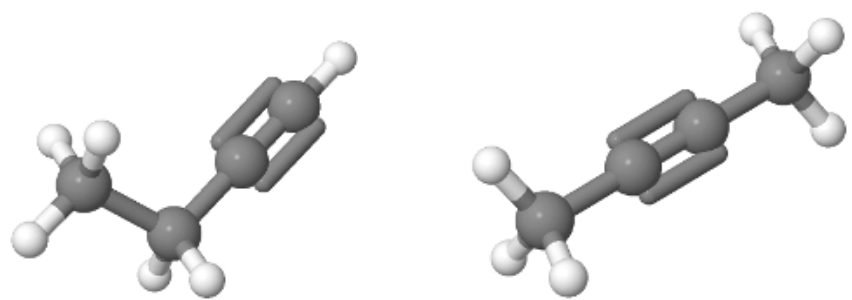

Figura 5: Ilustração dos dois alcinos isoméricos de fórmula molecular $\mathrm{C}_{4} \mathrm{H}_{6}$, o 1-butino, e o 2-butino. Figuras geradas por [2].

Finalmente, os cicloalcinos também são hidrocarbonetos insaturados que contêm um único ciclo em sua estrutura molecular, ao qual podem estar ligadas uma ou mais ramificações. Para esses compostos, a relação fixa entre o número de átomos de carbono e o de hidrogênio é $\mathrm{C}_{\mathrm{n}} \mathrm{H}_{2 \mathrm{n}-4}$. Como a ligação carbono-carbono tripla exige que haja uma natureza linear para os átomos de carbono que a cercam (isso é ilustrado pela estrutura molecular do 2-butino, mostrada na Figura 5), a estrutura molecular dos cicloalcinos pode ser altamente tensionada. Isso faz que só sejam estáveis cicloalcinos com um número grande de átomos de carbono, sendo que o ciclo-octino, $\mathrm{C}_{8} \mathrm{H}_{12}$, é o menor desses hidrocarbonetos que é estável, podendo ser isolado e armazenado (veja a Figura 6 e note como as ligações carbono-carbono ao redor da ligação tripla não são lineares e, portanto, são tensionadas). 


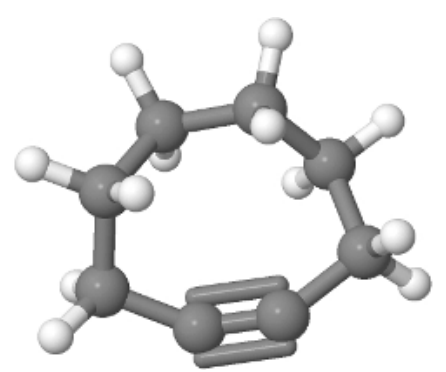

Figura 6: Ilustração do menor cicloalcino estável, o ciclo-octino. Figura gerada por [2].

Esses diferentes tipos de hidrocarbonetos insaturados podem ser hidrogenados, parcial ou totalmente. Por exemplo, o ciclo-octino pode ser hidrogenado parcialmente a ciclo-octeno $\left(\mathrm{C}_{8} \mathrm{H}_{14}\right.$, um cicloalceno) ou ainda a ciclo-octano $\left(\mathrm{C}_{8} \mathrm{H}_{16}\right.$, um cicloalcano); caso ocorra abertura de anel e hidrogenação máxima, obter-se-á o octano $\left(\mathrm{C}_{8} \mathrm{H}_{18}\right.$, um alcano acíclico), um dos componentes da gasolina.

\section{Modelagem matemática de hidrocarbonetos usando grafos}

A estrutura combinatória de um hidrocarboneto consiste das seguintes informações: (i) o número de átomos de carbono; (ii) o número de átomos de hidrogênio; (iii) qual átomo está ligado com qual, e quantas vezes.

Essa estrutura é modelada por um objeto matemático denominado grafo. O grafo deve ter um conjunto de pontos, em número igual à soma dos números de átomos de carbono e hidrogênio, e linhas ligando pontos que informam qual átomo está ligado com qual. Na Tabela 1 vemos exemplos de alcanos e de grafos que os modelam.

\begin{tabular}{|c|c|c|}
\hline nome & modelo espacial & modelo como grafo \\
\hline pentano & & - \\
\hline isopentano & & \\
\hline neopentano & & \\
\hline
\end{tabular}

Tabela 1: Modelos do pentano, isopentano e do neopentano, moléculas da família $\mathrm{C}_{\mathrm{n}} \mathrm{H}_{2 \mathrm{n}+2}$, com $\mathrm{n}=5$, apresentadas na Figura 1 . 
O grafo de uma molécula fornece-nos apenas informações combinatórias. Ele não descreve como os átomos colocam-se no espaço. Em particular, os ângulos entre as ligações não estão necessariamente retratados no grafo. Assim sendo, dois estereoisômeros do mesmo isômero não são distinguíveis por seu grafo. Na Tabela 2 vemos modelos de alcenos. O cis-2-buteno e o trans-2-buteno são estereoisômeros do mesmo isômero, e têm o mesmo grafo, pois sua constituição combinatória é a mesma (poderíamos desenhar o grafo do trans-2-buteno de outra forma, refletindo verticalmente a parte direita do desenho, mas o grafo seria essencialmente o mesmo, mudando apenas o desenho).

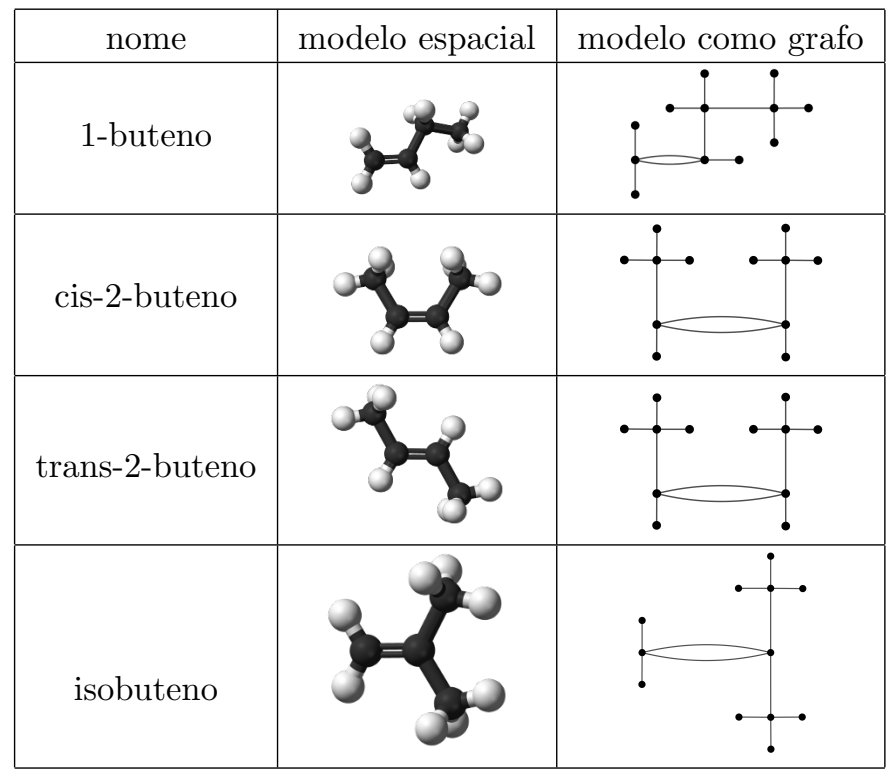

Tabela 2: Modelos do 1-buteno, do cis-2-buteno, do trans-2-buteno e do isobuteno, moléculas da família $\mathrm{C}_{\mathrm{n}} \mathrm{H}_{2 \mathrm{n}}$, com $\mathrm{n}=4$, apresentadas na Figura 3 .

Se um grafo modela um hidrocarboneto, os comprimentos das linhas que ligam pontos não correspondem, necessariamente, às distâncias relativas entre os átomos (referidas como comprimentos de ligação). Escolhemos esses comprimentos de modo a facilitar a leitura do grafo e deixar patentes as propriedades combinatórias. Em um tal grafo é fácil distinguir os pontos que representam os átomos de carbono daqueles que representam os de hidrogênio: basta contar o número de ligações que concorrem ao ponto. Pontos com quatro ligações representam átomos de carbono, e, com uma, átomos de hidrogênio.

Os grafos podem ser desenhados com pontos e linhas coloridas, como se vê na Figura 7. Fica mais bonito e pode facilitar a visualização, mas nada acrescenta de informação sobre as propriedades combinatórias da molécula. 

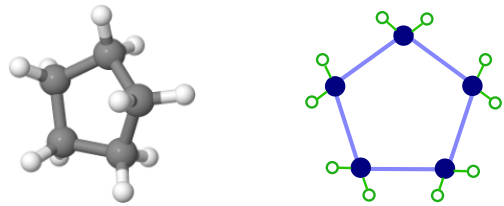

Figura 7: Ilustração da molécula do ciclopentano e um desenho "artístico" do grafo correspondente. Figura espacial gerada por [2]

Os grafos podem também modelar hidrocarbonetos cíclicos, como se vê na Figura 7 e na Tabela 3.

\begin{tabular}{|c|c|c|}
\hline nome & modelo espacial & modelo como grafo \\
\hline ciclobuteno & & 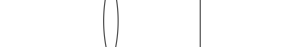 \\
\hline 1-metilciclopropeno & & ! \\
\hline 3-metilciclopropeno & & \\
\hline metilenociclopropano & & t \\
\hline
\end{tabular}

Tabela 3: Modelos do ciclobuteno, do 1-metilciclopropeno, do 3-metilciclopropeno e do metilenociclopropano, moléculas isoméricas, apresentadas na Figura 4.

Em Matemática, quando modelamos um fenômeno natural com um objeto geométrico (ou de outra natureza matemática), sempre pretendemos obter informações sobre esse fenômeno. Uma propriedade que buscamos alcançar ao modelar hidrocarbonetos $\mathrm{C}_{n} \mathrm{H}_{\mathrm{h}}$ com grafos é verificar as fórmulas usadas na Química. Por exemplo, nos alcanos acíclicos temos $h=2 n+2$, qualquer que seja o arranjo dos átomos. Em um alcano essa propriedade pode ser visualmente verificada quando os átomos de carbono estão dispostos "linearmente", como se vê na Figura 8. Observamos facilmente que, se o número de átomos de carbono for n, o de átomos de hidrogênio é $2 \mathrm{n}+2$. 


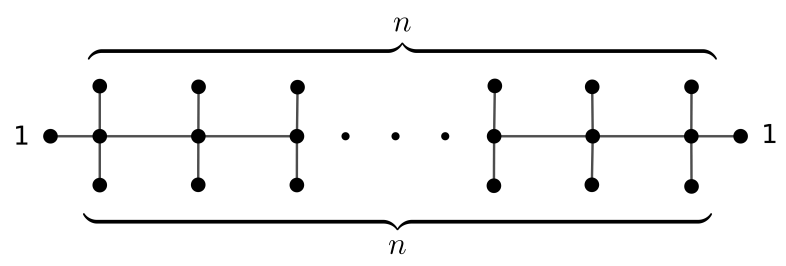

Figura 8: Representação como grafo da molécula de um alcano acíclico com n átomos de carbono em disposição linear.

Entretanto, os átomos de carbono podem ocorrer com outros arranjos. Por exemplo, se $\mathrm{n}=5$, vimos, na Tabela 1, três arranjos possíveis, isômeros da família de alcanos acíclicos cuja fórmula molecular é $\mathrm{C}_{5} \mathrm{H}_{12}$. Em Química, hidrocarbonetos com o mesmo número de átomos de carbono mas representados por grafos diferentes são exemplos de isômeros estruturais. Em todos esses arranjos continua valendo a fórmula $h=2 n+2$. No caso $n=5$ podemos constatar isso examinando cada desenho, mas, em geral, o número de árvores diferentes aumenta consideravelmente com n. Na próxima seção, veremos como algumas propriedades combinatórias dos grafos permitem-nos demonstrar essas fórmulas.

\section{Definição de grafos e alguns teoremas de contagem}

Dentre as várias maneiras de definir um grafo, escolhemos aquela que é mais conveniente para o uso que faremos neste texto.

Um grafo é um conjunto finito de pontos, denominados vértices, reunido com um conjunto finito de linhas que ligam pares (não ordenados) desses vértices, chamadas arestas. Apenas as extremidades de uma aresta são vértices (e são vértices diferentes). Arestas que ligam o mesmo par de vértices são chamadas arestas múltiplas. O grau g(A) de um vértice A é o número de arestas que a ele concorrem.

Indicamos os vértices por $\mathrm{A}, \mathrm{B}, \mathrm{C}, \ldots$, ou por essas letras indexadas, como $\mathrm{A}_{1}, \mathrm{~A}_{2}, \ldots$ Analogamente indicamos as arestas por a, b, c, ..., ou por essas letras indexadas.

As relações entre vértices e arestas de um grafo podem ser definidas através de um desenho, como os que vemos na Figura 9. Nossos desenhos são planares, e fica entendido que não consideramos relevantes os pontos de interseção de arestas que não sejam vértices (em outros contextos podem ser importantes). No grafo $\mathcal{G}_{2}$ da Figura 9 vemos um exemplo de arestas múltiplas, $c_{1}$ e $c_{2}$. Na mesma figura, em $\mathcal{G}_{1}$ temos $\mathrm{g}(\mathrm{D})=3$ e em $\mathcal{G}_{2}$ temos $\mathrm{g}(\mathrm{D})=4$.
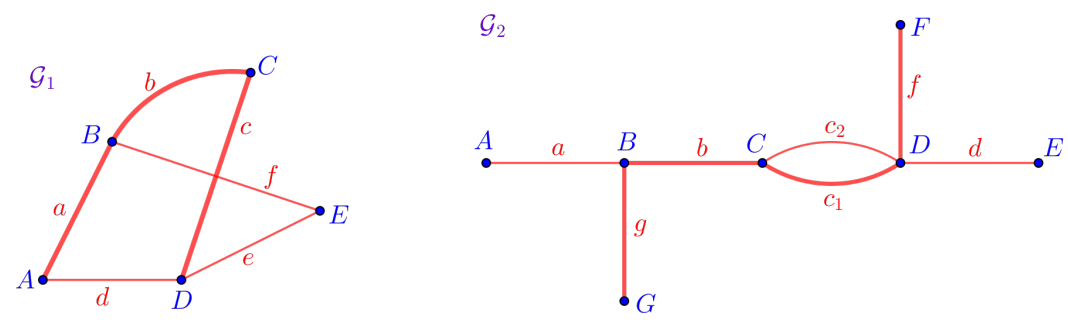

Figura 9: Exemplos de grafos.

Um caminho em um grafo é uma lista ordenada de vértices e arestas $A_{1}, a_{1}, A_{2}, a_{2}, \ldots, a_{n-1}, A_{n}$, 
de forma que $A_{i}$ e $A_{i+1}$ são os extremos de $a_{i}, 1 \leq i \leq n-1$. Os vértices $A_{1}$ e $A_{n}$ são os extremos do caminho. $\mathrm{O}$ caminho se diz fechado quando $\mathrm{A}_{1}=\mathrm{A}_{\mathrm{n}}$. Uma trilha é um caminho em que as arestas são diferentes duas a duas. Um ciclo é uma trilha fechada em que os vértices são diferentes dois a dois, exceto os extremos. Um grafo diz-se cíclico quando tem ciclos, e acíclico quando não tem ciclos. Um grafo diz-se conexo quando, dados dois vértices quaisquer $\mathrm{A}$ e $\mathrm{B}$, existe um caminho com extremos A e B.

Na Figura 9 tanto o grafo $\mathcal{G}_{1}$ quanto o $\mathcal{G}_{2}$ são conexos. No grafo $\mathcal{G}_{1}$ está distinguida a trilha $\mathrm{AaBbCcD}$, e no grafo $\mathcal{G}_{2}$ está distinguida a trilha $\mathrm{GgBbCc}_{1} \mathrm{DfF}$. O grafo $\mathcal{G}_{1}$ tem vários ciclos, um deles é AaBfEeDdA. Em $\mathcal{G}_{2}$ temos o ciclo $\mathrm{Cc}_{1} \mathrm{Dc}_{2} \mathrm{C}$.

Mais detalhes e aplicações de grafos podem ser vistas em M. Goldbarg e E. Goldbarg (2012) [3], em E. L. Lima (1988) [6] e em D. B. West (2001) [7].

No presente texto estamos interessados em alguns resultados de contagem sobre os grafos. O primeiro é:

Teorema 1. Em qualquer grafo, a soma dos graus de todos os vértices é o dobro do número de arestas.

Demonstração. Dado um vértice, seu grau conta o número de arestas a ele ligadas. Assim, se somarmos todos os graus, contamos todas as arestas. Mas, nessa soma, cada aresta foi contada duas vezes, pois a cada aresta correspondem dois vértices. Portanto, a soma dos graus é o dobro do número de arestas.

Árvores são grafos conexos e acíclicos. Por serem grafos mais simples, fica fácil estudar algumas de suas propriedades.

Lema 1. Em um grafo com um único vértice não existem arestas.

Demonstração. Por definição de grafo.

Lema 2. Em toda árvore com dois vértices ou mais não existem arestas múltiplas.

Demonstração. Arestas múltiplas formam ciclos, mas árvores são acíclicas. Portanto, vale a afirmação.

Lema 3. Se uma árvore tem dois vértices, eles têm grau 1 e existe uma única aresta.

Demonstração. É uma consequência do Lema 2.

Lema 4. Se o grau de um vértice $\mathrm{A}$ de uma árvore for $\mathrm{g} \geq 1$, então existem $\mathrm{g}$ outros vértices $\mathrm{A}_{1}$, ..., $\mathrm{A}_{\mathrm{g}}$ (diferentes dois a dois) ligados com $\mathrm{A}$ por $\mathrm{g}$ arestas diferentes.

Demonstração. Se o grau de A é g $\geq 1$, por definição existem g arestas (diferentes duas a duas) que a ele concorrem. As segundas extremidades dessas arestas são vértices $A_{1}, \ldots, A_{g}$. Esses vértices são diferentes dois a dois pois, caso contrário, haveria arestas múltiplas, o que contraria o Lema 2. Concluímos que vale a afirmação. 
Proposição 1. Toda árvore com dois vértices ou mais tem pelo menos um vértice de grau 1.

Demonstração. Se a árvore tiver dois vértices, a afirmação segue do Lema 3. Suponhamos que tenha três vértices ou mais. Escolhemos um vértice qualquer e o chamamos de $A_{1}$. Se o grau de $A_{1}$ for 1 , terminamos. Suponhamos que o grau de $A_{1}$ seja $\geq 2$. Então, pelo Lema 4, existem dois vértices $A_{0}$ e $A_{2}$ (diferentes) e arestas a 01 ligando $A_{0}$ com $A_{1}$ e $a_{12}$ ligando $A_{1}$ com $A_{2}$. Se o grau de $A_{2}$ for 1 , terminamos. Suponhamos que o grau de $A_{2}$ seja $\geq 2$. Então existe um vértice $A_{3}$ e uma aresta a 23 ligando $A_{2}$ com $A_{3}$. Como a árvore é um grafo acíclico, $A_{3} \neq A_{i}, 1 \leq i \leq 2$. Continuando com o mesmo procedimento, no j-ésimo passo encontramos uma trilha $A_{1} a_{12} A_{2} a_{23} A_{3} \ldots A_{j+1}$ com $\mathrm{j}+1$ vértices, e verificamos se $A_{j+1}$ tem grau 1 ou $\geq 2$. Como o número de vértices é finito, em algum passo a resposta será grau 1, e, assim, terminamos.

Proposição 2. Toda árvore com $\mathrm{m} \geq 1$ vértices tem exatamente $\mathrm{m}-1$ arestas.

Demonstração. Usaremos o Método da Indução Completa sobre o número de vértices m. Vimos no Lema 1 que, se $\mathrm{m}=1$, a afirmação é verdadeira. Seja $\mathrm{m} \geq 2$, e suponhamos que a afirmação seja verdadeira para toda árvore com $\mathrm{m}-1$ vértices. Consideremos uma árvore qualquer com $\mathrm{m}$ vértices. Devido à Proposição 1 , ela tem um vértice de grau 1. Retirando esse vértice do grafo, e retirando a única aresta que a ele concorre, obtemos um novo grafo que ainda é uma árvore. Como essa árvore tem $\mathrm{m}-1$ vértices, vale para ela a afirmação, e, assim, ela tem $\mathrm{m}-2$ arestas. Logo, a árvore de $\mathrm{m}$ vértices dada tem $\mathrm{m}-1$ arestas, pois existe uma única aresta diferente nos dois grafos. Segue que a afirmação é verdadeira para toda árvore com $m \geq 1$ vértices.

Teorema 2. Em qualquer árvore com $\mathrm{m} \geq 1$ vértices, a soma dos graus de todos os vértices é $2(\mathrm{~m}-1)$.

Demonstração. Do Teorema 1 sabemos que a soma dos graus é o dobro das arestas. Se a árvore tem $\mathrm{m}$ vértices, a Proposição 2 nos diz que ela tem $\mathrm{m}-1$ arestas. Assim, ao somarmos os graus, obtemos o valor em dobro, isto é, $2(\mathrm{~m}-1)$.

Vejamos algumas aplicações desses resultados.

\section{Fórmula $\mathrm{C}_{n} \mathrm{H}_{2 \mathrm{n}+2}$ dos alcanos acíclicos}

O modelo em grafo de um alcano acíclico é uma árvore com vértices de grau 1 e vértices de grau 4.

Proposição 3. Se uma árvore tem $\mathrm{n} \geq 1$ vértices com grau 4 e os outros vértices têm grau 1 , então esses outros vértices são em número de $2 \mathrm{n}+2$.

Demonstração. Seja h o número de vértices de grau 1. Portanto, a soma dos graus é $4 \mathrm{n}+\mathrm{h}$. Como a árvore tem $n+h$ vértices, sabemos, da Proposição 2, que ela tem $n+h-1$ arestas. Como a soma dos graus conta as arestas em dobro, temos

$$
4 \mathrm{n}+\mathrm{h}=2(\mathrm{n}+\mathrm{h}-1)
$$

Resolvendo para $\mathrm{h}$, obtemos $\mathrm{h}=2 \mathrm{n}+2$, que é a afirmação proposta. 
Concluímos com o

Teorema 3. A fórmula geral dos alcanos acíclicos é $\mathrm{C}_{\mathrm{n}} \mathrm{H}_{2 \mathrm{n}+2}$.

Demonstração. Todo alcano acíclico tem como modelo uma árvore com vértices de grau 4 (pelo menos um) e vértices de grau 1. Portanto, o resultado segue da Proposição 3.

Exemplos de alcanos acíclicos e seus grafos estão apresentados na Tabela 1.

\section{Fórmula $\mathrm{C}_{n} \mathrm{H}_{2 n}$ dos cicloalcanos e dos alcenos}

Vejamos agora dois resultados para grafos com um único ciclo (cicloalcanos e alcenos):

Proposição 4. Se um grafo conexo com um único ciclo tem $\mathrm{m}$ vértices, então: (i) tem $\mathrm{m}$ arestas; (ii) a soma dos graus é $2 \mathrm{~m}$.

Demonstração. Seja $\mathcal{G}$ o grafo dado, e seja $\ell$ o número de suas arestas. Construímos um novo grafo $\mathcal{H}$ da seguinte forma: excluímos uma aresta do ciclo de $\mathcal{G}$ e em cada um de seus extremos acrescentamos uma nova aresta com um novo vértice. Assim, $\mathcal{H}$ tem $\mathrm{m}+2$ vértices e $\ell+1$ arestas. Mas $\mathcal{H}$ é uma árvore, pois continua sendo conexo e agora é acíclico. Sabemos da Proposição 2 que $\ell+1=(\mathrm{m}+2)-1$. Segue que $\ell=\mathrm{m}$, e fica verificada a afirmação (i). A afirmação (ii) segue diretamente do Teorema 1.

Para melhor acompanharmos a demonstração da Proposição 4, a Figura 10 traz um exemplo de como se transforma um grafo conexo com um ciclo em outro conexo acíclico.
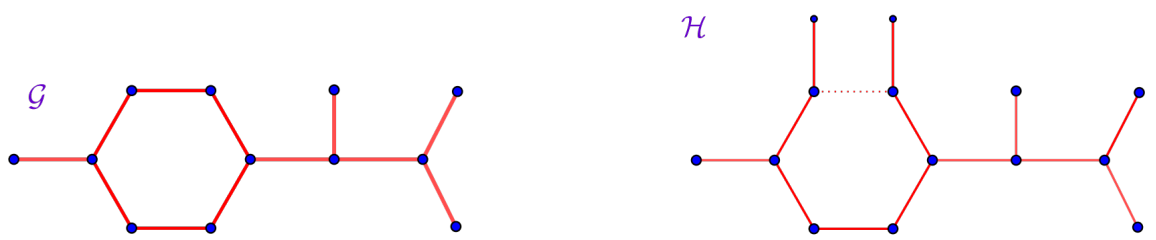

Figura 10: Operação de corte em um grafo. Nesse caso, uma operação geométrica, mas que tem ligação com a química de hidrocarbonetos (vide abaixo).

Corolário 1. Se um grafo conexo com um único ciclo tem n vértices de grau 4 e o restante de grau 1 , então esses são em número de $2 \mathrm{n}$.

Demonstração. Seja h o número de vértices de grau 1. Então a soma dos graus é $4 \mathrm{n}+\mathrm{h}$. Por outro lado, esse grafo tem $n+h$ vértices, e a Proposição 4 diz que essa soma é também $2(n+h)$. Assim,

$$
4 n+h=2(n+h)
$$

Resolvendo para $\mathrm{h}$ temos $\mathrm{h}=2 \mathrm{n}$, que é a afirmação proposta.

Concluímos com o

Teorema 4. A fórmula geral dos cicloalcanos e dos alcenos é $\mathrm{C}_{\mathrm{n}} \mathrm{H}_{2 \mathrm{n}}$. 
Demonstração. Os cicloalcanos e os alcenos são modelados por grafos com um único ciclo, com vértices de grau 4 (pelo menos dois) e o restante de grau 1. Portanto, o resultado segue do Corolário 1.

Observamos que a operação de corte realizada acima é puramente geométrica, e pode ser feita em qualquer grafo. Mas, assim como a arte imita a vida, a Matemática também imita a natureza. Nos hidrocarbonetos, essa operação corresponde à hidrogenação, comentada no final da Seção 3 e no final da Seção 4. Na Figura 11 vemos que o metilciclopropano $\left(\mathrm{C}_{4} \mathrm{H}_{8}\right)$ pode ser hidrogenado, obtendo-se o butano $\left(\mathrm{C}_{4} \mathrm{H}_{10}\right)$. Na Figura 12 vemos que essa operação química corresponde à operação geométrica que denominamos "corte", com a qual transformamos o grafo que modela o metilciclopropano na árvore que modela o butano.

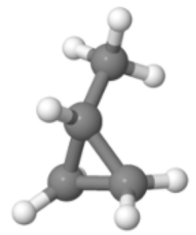

hidrogenação

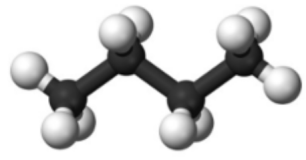

Figura 11: Hidrogenação do metilciclopropano em butano, um dos possíveis produtos da reação de hidrogenação.
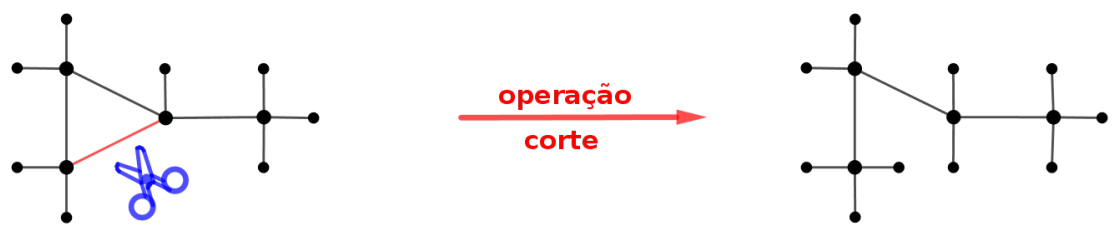

Figura 12: Operação de corte no grafo do metilciclopropano, da qual resulta o grafo do butano.

\section{Fórmula geral dos hidrocarbonetos}

O procedimento de contagem da Seção 8 pode ser generalizado. Seja $\mathcal{G}$ um grafo conexo com $\mathrm{m}$ vértices e $\ell$ arestas, e contendo ciclos. Suponhamos que com r operações de corte obtenhamos uma árvore. Ela tem $\mathrm{m}+2 \mathrm{r}$ vértices e $\ell+\mathrm{r}$ arestas. Portanto, $\ell+\mathrm{r}=\mathrm{m}+2 \mathrm{r}-1 \Rightarrow \ell=\mathrm{m}+\mathrm{r}-1$. Assim, a soma dos graus de $\mathcal{G}$ é $2 \ell=2(\mathrm{~m}+\mathrm{r}-1)$.

Agora, se $\mathcal{G}$ tem $\mathrm{n}$ vértices de grau 4 e os outros $\mathrm{h}$ vértices de grau 1 , temos $\mathrm{m}=\mathrm{n}+\mathrm{h}$ e a soma dos graus é $4 n+h$. Portanto, $4 n+h=2(n+h+r-1)$. Resolvendo para h vem $h=2 n-2 r+2$.

Em geral temos:

Teorema 5. Seja $\mathcal{G}$ um grafo com $\mathrm{m}$ vértices e $\ell$ arestas e suponhamos que $\mathrm{r}$ operações de corte transformem o grafo em uma árvore. Então: (i) $\ell=\mathrm{m}+\mathrm{r}-1$, (ii) a soma dos graus de $\mathcal{G}$ é $2 \ell=2(\mathrm{~m}+\mathrm{r}-1)$; (iii) se $\mathcal{G}$ tem $\mathrm{n}$ vértices de grau 4 e $\mathrm{h}$ de grau 1 (e nenhum outro), então $\mathrm{h}=2 \mathrm{n}-2 \mathrm{r}+2$. 
Corolário 2. Suponhamos que um hidrocarboneto, com $\mathrm{n}$ átomos de carbono, seja modelado por um grafo tal que sejam necessárias $\mathrm{r}$ operações de corte para transformá-lo em uma árvore. Então a fórmula do hidrocarboneto é $\mathrm{C}_{\mathrm{n}} \mathrm{H}_{2 \mathrm{n}-2 \mathrm{r}+2}$.

Demonstração. Segue do resultado do Teorema 5, parte (iii).

Vejamos um primeiro exemplo. Os cicloalcenos (confira exemplos na Figura 4) têm um ciclo formado por três ou mais átomos de carbono, sendo que no ciclo existe uma ligação dupla entre dois átomos de carbono. São necessárias duas operações de corte para transformar seus grafos em árvores. Tomando por base a notação do Corolário 2, temos $\mathrm{r}=2$. Assim, se $\mathrm{n}$ é o número de átomos de carbono, o de hidrogênio é $\mathrm{h}=2 \mathrm{n}-2 \mathrm{r}+2=2 \mathrm{n}-2 \cdot 2+2=2 \mathrm{n}-2$. Portanto, essas moléculas têm a fórmula geral $\mathrm{C}_{\mathrm{n}} \mathrm{H}_{2 \mathrm{n}-2}$. Para $\mathrm{n}=4$, a fórmula molecular é $\mathrm{C}_{4} \mathrm{H}_{6}$, que é o caso dos três cicloalcenos isoméricos mostrados na Figura 4.

Vejamos um segundo exemplo. Embora o benzeno não seja um hidrocarboneto, podemos ver que a ele aplica-se a operação de corte. Ele é constituído por um ciclo hexagonal, com 6 átomos de carbono. Em sua forma canônica (estrutura de Kekulé), das seis ligações entre esses átomos, três são duplas, em arestas alternadas do hexágono. Na Figura 13, desenho da esquerda, vemos o grafo da forma canônica do benzeno. Na verdade, essas ligações duplas movimentam-se nas seis arestas, devido ao fenômeno da ressonância, mas nos ateremos à aproximação representada pela forma canônica.
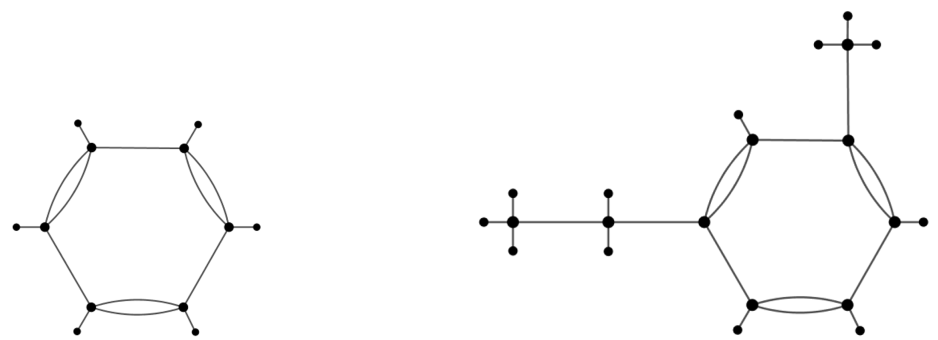

Figura 13: Grafos das formas canônicas do benzeno e do 1-etil-3-metilbenzeno.

Para transformar o grafo da forma canônica do benzeno em uma árvore, precisamos de $\mathrm{r}=4$ cortes. Como essa molécula tem $\mathrm{n}=6$ átomos de carbono, pelo Corolário 2 o número de átomos de hidrogênio é $\mathrm{h}=2 \mathrm{n}-2 \mathrm{r}+2=2 \cdot 6-2 \cdot 4+2=6$. Portanto, a fórmula molecular do benzeno é $\mathrm{C}_{6} \mathrm{H}_{6}$, o que pode ser verificado diretamente no grafo.

O benzeno é a base da família dos compostos aromáticos monocíclicos, que têm um anel benzênico. Se o número de átomos de carbono for $\mathrm{n}$, o de hidrogênio é $\mathrm{h}=2 \mathrm{n}-2 \mathrm{r}+2=2 \mathrm{n}-2 \cdot 4+2=2 \mathrm{n}-6$. A fórmula geral dessa família é $\mathrm{C}_{\mathrm{n}} \mathrm{H}_{2 \mathrm{n}-6}$. Um exemplo é o 1-etil-3-metilbenzeno, cujo grafo está desenhado na Figura 13, à direita. Sua fórmula é $\mathrm{C}_{9} \mathrm{H}_{12}$.

\section{Observações sobre contagem de isômeros}

Dados $\mathrm{m}$ pontos, quantos grafos diferentes podem ser construídos? Os $\mathrm{m}$ pontos (considerados distinguíveis) podem formar $\left(\begin{array}{c}\mathrm{m} \\ 2\end{array}\right)$ pares (não ordenados). Ao constituir um grafo, para cada par 
de pontos temos duas escolhas: ligar ou não ligar. Usando o princípio multiplicativo vemos que podemos construir

$$
2^{\left(\begin{array}{c}
m \\
2
\end{array}\right)}
$$

grafos.

Um interesse particular aqui é contar as árvores. Um teorema de Cayley de 1889 [Arthur Cayley, 1821-1895] diz-nos que existem

$$
\mathrm{m}^{\mathrm{m}-2}
$$

árvores. Ao contar árvores com $m$ vértices, são assignados, a cada vértice, todos os possíveis graus $1 \leq \mathrm{g} \leq \mathrm{m}-1$, de modo que a soma dos graus seja $2(\mathrm{~m}-1)$. Quatro demonstrações diferentes desse resultado podem ser lidas em M. Aigner e G. M. Ziegler (2018) [1], a partir da pág. 235.

As árvores que modelam alcanos acíclicos obedecem a algumas restrições. Elas correspondem a árvores com $\mathrm{m}$ vértices, dos quais existem $\mathrm{n}$ de grau $4 \mathrm{e} h=2 \mathrm{n}+2$ de grau 1 , de forma que $\mathrm{n}+\mathrm{h}=\mathrm{m}$. Além disso os vértices de mesmo grau são indistinguíveis. A literatura informa que não é conhecida uma fórmula de contagem para esse caso especial. É possível realizar o cálculo através de algoritmos recursivos ou usando o princípio de enumeração de Pólya-Redfield [George Pólya, 1887-1985, John H. Redfield, 1879-1944]. Maiores explicações, incluindo uma história sobre o assunto, podem ser lidas em D. J. Klein (2002) [5].

Para o leitor ter uma ideia do número de árvores diferentes, possíveis modelos de alcanos, transcrevemos, na Tabela 4, alguns valores obtidos de I. Gutman (2008), pág. 55 [4]. Nessa tabela, n é o número de vértices que correspondem aos átomos de carbono, e A é o número de árvores diferentes.

\begin{tabular}{|c|c|c|c|c|c|c|c|c|c|c|c|c|c|c|}
\hline $\mathrm{n}$ & 1 & 2 & 3 & 4 & 5 & 6 & 7 & 8 & 9 & 10 & $\ldots$ & 20 & $\ldots$ & 30 \\
\hline $\mathrm{A}$ & 1 & 1 & 1 & 2 & 3 & 5 & 9 & 18 & 35 & 75 & $\ldots$ & 366319 & $\ldots$ & 4111846763 \\
\hline
\end{tabular}

Tabela 4: Número A de árvores diferentes, possíveis modelos de alcanos, em função do número $\mathrm{n}$ de átomos de carbono.

Vemos que o número de árvores diferentes aumenta exponencialmente com n. Observamos que esses números são teóricos e dizem respeito a uma propriedade matemática. Nem toda árvore tem composto químico correspondente conhecido. Na realidade, à medida que $\mathrm{n}$ aumenta, diminui a probabilidade de tais moléculas químicas existirem e, portanto, diminui muito o número $\mathrm{A}$ de árvores que correspondem efetivamente a compostos químicos.

\section{Agradecimentos}

Agradece-se apoio da Capes - Coordenação de Aperfeiçoamento de Pessoal de Nível Superior (código de financiamento 001) e bolsa do CNPq - Conselho Nacional de Desenvolvimento Científico e Tecnológico (processo no $311970 / 2017-6$ ).

\section{Referências}

[1] Aigner, M. and Ziegler, G.M. Proofs from the Book. $6^{\mathrm{a}}$ edição. Berlin, Springer, 2018. https: //doi.org/10.1007/978-3-662-57265-8 
[2] Chemapps: aplicativo de geração de figuras espaciais de elementos químicos. Disponível em https://chemapps.stolaf.edu/jmol/ Consultado em 24 de julho de 2020.

[3] Goldbarg, M. e Goldbarg E. Grafos: Conceitos, Algoritmos e Aplicações. Rio de Janeiro: Elsevier, 2012.

[4] Gutman, I. "The Chemical Formula $\mathrm{C}_{\mathrm{n}} \mathrm{H}_{2 \mathrm{n}+2}$ and its Mathematical Background". The Teaching of Mathematics 2008, Vol. XI, 2, pp. 53-61.

[5] Klein, D. J., Babica, D. e Trinajstica, N. "Enumeration in Chemistry". In: Hinchliffe, A. Chemical Modelling: Applications and Theory. Volume 2, Capítulo 2. Cambridge: The Royal Society of Chemistry, 2002. p. 56-95.

[6] Lima, E. L. "Alguns Problemas clássicos sobre Grafos". Revista do Professor de Matemática,

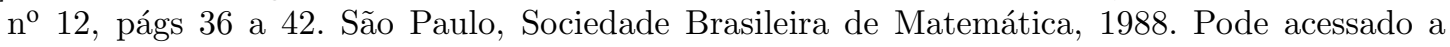
partir do endereço http://rpm.org.br/BuscaAvancada.aspx.

[7] West, D. B. Introduction to Graph Theory. Second Edition. Upper Saddle River: Prentice Hall, 2001.

[8] Autores: Ben Mills e Jynto. Data: 2 de janeiro de 2010 Copyright: domíno público. Disponível em https://commons.wikimedia.org/wiki/File:Pentane-3D-balls.png e /File:Isopentane-3D-balls.png e /File:Neopentane-3D-balls.png Consultado em 28 de julho de 2020.

[9] Autor: Ben Mills. Copyright: domíno público. Disponível em https://commons.wikimedia.org/ wiki/File:But-1-ene-3D-balls.png (data:10 de janeiro de 2008), /File:Cis-but-2-ene-3D-balls.png (data: 15 de maio de 2007), /File:Trans-but-2-ene-3D-balls.png (data: 16 de maio de 2007) e /File:Isobutylene-3D-balls.png (data: 11 de janeiro de 2008). Consultado em 28 de julho de 2020.

[10] Wikipedia Alkane. Cycloalkane. Alkene. Disponível em https://en.wikipedia.org/wiki/Alkane e /Cycloalkane e /Alkene Consultado em 28 de outubro de 2019.

Roberto Ribeiro Paterlini Universidade Federal de São Carlos <paterlini@ufscar.br>

Romeu C. Rocha-Filho Universidade Federal de São Carlos <romeu@ufscar.br>

Recebido: 13/08/2021 Publicado: 18/11/2021 